\title{
Sensor Network Node Positioning and Fusion Algorithm of Electric Vehicle Charging Stood in Cloud Computing
}

\author{
Xu Lijuan ${ }^{1}$, Liu Dalong ${ }^{2}$ \\ ${ }^{1}$ Huashang College Guangdong University of Finance \& Economics, Department of Information \\ Engineering, Guangdong Zengcheng 511300, China; \\ ${ }^{2}$ Department of Mechanical and Electrical and Information Engineering, Huali College of \\ Guangdong University of Technology, Guangdong Zengcheng 511325,China \\ yilanwoxin@126.com
}

Keywords: electric vehicles; charging station; wireless sensor network; localization

\begin{abstract}
In the cloud computing environment, the sensor net model of electric vehicle charging station is designed, the fusion and localization algorithm of sensor network nodes are researched, the mobile terminal is used to realize the quickly localization and searching for electric vehicle charging station, charging station can be localized, and improve service function. A fusion and positioning algorithm of sensor network nodes is proposed based on strong tracking distance estimation. The station sensor network channel estimation and modulation model of electric vehicle charging is constructed, in the cloud computing environment, stochastic resonance fusion technology is taken to realize the accurate positioning and data fusion of WSN nodes. The station structure model of electric vehicle charging is obtained, and the multi-sensor data fusion filtering is realized. The noise suppression is obtained, and the state estimation and information value are calculated. The method of tracking filter taken for distance estimation, the charging fusion and positioning of station nodes is obtained. The experiment results show that the accuracy of station sensor network node distance estimation and fusion positioning is high, and it has superior performance in application.
\end{abstract}

\section{Introduction}

Because the green travel concept is proposed deeply, and due to the energy saving and environmental protection requirement, the electric car gradually come into people's lives. The cars of electric vehicles take the fuel battery as the power supply, by charging the energy supplement is realized, it has the green environmental protection and convenience features. However, the charging of electric vehicles is not convenient compare to the fuel automobile, it also become the difficulties and restrict of the development of electric vehicles, the optimization and search node positioning method of electric vehicle charging pile network is researched. It can be convenient for driver to realize fast searching and location of charging stations, and provide useful information on the mobile terminal equipment, to solve the vehicle actual use in the process of rapid positioning. Therefore, in the cloud computing environment, the design of electric vehicle charging station sensor net model of charging station is obtained. Fusion localization algorithm of sensor network node is researched. It has become a hot topic of current EV energy supply and communication network model construction[1].

In the traditional method, multi neural network self organizing map control method is used to realize the computing environment of electric vehicle charging station sensor net node positioning and data fusion, the self sensor network node and distributed control is realized[2]. The reference [3] proposes an adaptive localization algorithm for wireless sensor network node localization based on FPGA, the detection and localization of sensor network nodes are realized, and the multi sensor fusion is designed for sensor network detection, the node searching is realized, but the algorithm needs RFID technology for automatic identification, and the practical application has little value. In the reference [4], the improved sliding mode control algorithm is proposed, and the network security routing algorithm is proposed based on credibility analysis, the model of cloud computing 
is constructed, fusion localization algorithm of electric vehicle charging station in the sensor network is improved, and the nonlinear control has good performance, but it cannot solve the node location and search problems of random distribution characteristics, and the distribution of nodes appear in bad condition in sensor networks, and computing cost is big, the compute is complex, the algorithm is difficult to implement. Aiming at these problems, study and explore how to use the mobile terminal to quickly locate and search for electric vehicle charging station, network data transmission is optimized, electric vehicle charging station sensor net node fusion and positioning are researched, A fusion and positioning algorithm of sensor network nodes is proposed based on strong tracking distance estimation. The station sensor network channel estimation and modulation model of electric vehicle charging is constructed, in the cloud computing environment, stochastic resonance fusion technology is taken to realize the accurate positioning and data fusion of WSN nodes[5,6]. The station structure model of electric vehicle charging is obtained, and the multi-sensor data fusion filtering is realized. The noise suppression is obtained, and the state estimation and information value are calculated. The method of tracking filter taken for distance estimation, the charging fusion and positioning of station nodes is obtained. The experiment results show that the accuracy of station sensor network node distance estimation and fusion positioning is high, and it has superior performance in application. In the end, the simulation experiment is taken, and performance analysis and verification are presented, the effective conclusions are formed in this paper.

\section{Architecture model of electric vehicle charging station sensor network}

A problem description and Wireless Sensor Networks model. In order to realize the rapid positioning and search of electric vehicle charging stations with mobile terminal equipment, it can provide useful information on the mobile terminal equipment to solve the actual use of electric vehicles, the rapid positioning can be realized in the process of implementation, the navigation and communication of filling station and charging pile are realized. It needs to build a model of wireless sensor networks. In the multi-sensor system, each sensor usually has different measuring characteristics, the connected graph $G$ and $V$ show the network node sets, $V^{2}$ shows the of arbitrary two-point boundary set, set space between the sensor nodes is $d$. Assumed each sensor is independent of each other, a sensor network is composed of a plurality of nodes in a sensor network deployed in the monitoring region, through the optimization of nodes in wireless sensor network, the deployment of tracking system is designed, perception and localization of network system, perception, acquisition and processing of network information of object are realized in the coverage area.

The sensor network node positioning and fusion model of electric vehicle charging station sensor network can be expressed as:

$$
z_{k}^{i}=h_{k}^{i}\left(x_{k}, u_{k}\right)+v_{k}^{i}, i=1,2, \ldots, M
$$

In the formula, $i(i=1,2, \ldots, M)$ is the parameter corresponding to the sensor (e.g. $z_{k}^{i}$ is the measurement vector in the moment of $\left.k_{\text {the sensor }}{ }^{i}\right), v^{i}$ is the merging noise of relay neuron control sensor network node ${ }^{i}$, and $E\left[v_{k}^{i}\left(v_{j}^{i}\right)^{T}\right]=R_{k}^{i} \delta_{k j}, E\left[w_{k}\left(v_{j}^{i}\right)^{T}\right]=0$; considering the needs of sensor network node location and data monitoring, assuming the sensor node distribution model of network system is:

$$
\frac{d h_{T h}}{d t}=\frac{\left(h_{\infty}\left(v_{T h}\right)-h_{T h}\right)}{\tau_{h}\left(v_{T h}\right)} \frac{d w_{T h}}{d t}=\frac{\left(w_{\infty}\left(v_{T h}\right)-w_{T h}\right)}{\tau_{w}\left(v_{T h}\right)}
$$

In the cloud computing environment, the node interval dominant point set is:

$$
D_{2}(u)=N_{2}(u) \cap M I S
$$

Station sensor networks generates a random failure in the initial time in random, the connection plug-in electric vehicles is analyzed, , in the next time period, it gets the electric vehicle charging 
station network overload sensor edges, ${ }_{i j}\left(e_{i j} \geq 0\right)$ an edge node types $E_{i}$ for fusion of the station random sensor network communication, the electric vehicle charging is realized, and $a n n\left(u, r_{1}, r_{2}, \alpha\right)$ is used to show the orthogonality of deflection vector at node u inelectric vehicle charging station network, each node is assigned a time slice, charging cross orthogonal deflection angle is $\alpha$, communicate in the annular region radius is formed between ${ }^{r_{1}}$ and ${ }^{r_{2}}$. The sensing network is solved through gateway access Internet solutions based on the mobile terminal and RFID equipment, the two bioavailability combination model for network channel are:

$$
\bar{C}_{i j}=\sum_{\tau}\left(x_{i \tau}-\bar{x}_{i \tau}\right)\left(x_{j \tau}-\bar{x}_{j \tau}\right) \quad \tilde{C}_{i j}=\sum_{\tau}\left(x_{i \tau}-\tilde{x}_{i \tau}\right)\left(x_{j \tau}-\tilde{x}_{j \tau}\right)
$$

The electric vehicle charging construction and communication model of sensor network model station is realized, and it can provide the model base for fusion positioning of sensor network node.

B Channel estimation and modulation model of electric vehicle charging station sensor network. In the construction of electric vehicle charging station sensor network based on the above model, channel estimation and channel modulation are carried out, according to the remaining degree rule, the descendant node failure overload can be expressed as:

$$
e_{i j}=p_{1}(i) \cdot p_{2}(j+1) \cdot p_{3}(i j)
$$

In the cloud computing environment, assuming that the electric vehicle charging sensor network $I_{S M}$ is the current input channel station sensor network for perceived estimates, its performance is periodic and intermittent in random, the basal nucleus of abnormal periodic square wave simulation is:

$$
I_{S M}=A_{S M} H\left(\sin \left(\frac{2 \pi t}{\rho_{S M}}\right)\right)\left(1-H\left(\sin \left(\frac{2 \pi t+D_{S M}}{\rho_{S M}}\right)\right)\right)
$$

Where, $A_{S M}$ is the amplitude square wave signal, $\rho_{S M}$ is the cycle of sensor network node distribution of the square wave signal, the sensor network base station collects all the data nodes, automatic control is realized, the fusion data is sent to the node $\mathrm{K}$, node data monitoring is taken. In the cloud computing environment, based on stochastic resonance fusion technology, realize the accurate positioning of the nodes in a sensor network and data fusion. The diagram is shown in Figure 1.

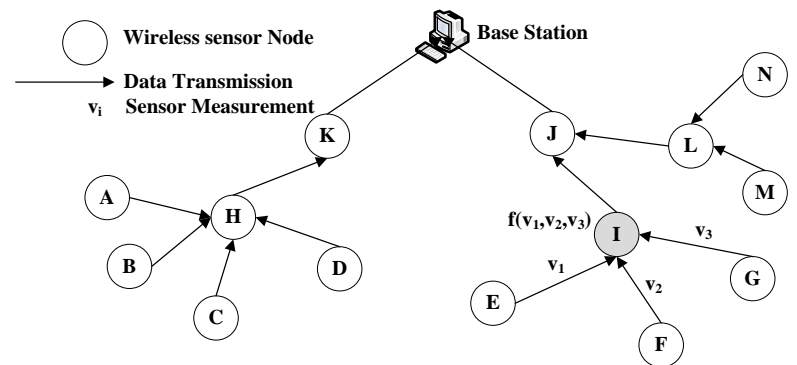

Fig. 1 Accurate positioning and data fusion diagram of charging station sensor net node

In Figure 1, the periodic square wave energy is set in the sensor network node distribution conditions, $I_{S M}$ is replaced as $V_{S M}$, random overload edge is calculated, in the channel network communication system based on wireless sensor network, channel estimation and modulation are taken, and the estimation results are expressed as:

$$
\begin{gathered}
x(k+1)=A(k) x(k)+\Gamma(k) w(k) \\
z_{i}(k)=H_{i}(k) x(k)+u_{i}(k), \quad i=1,2, \cdots, N
\end{gathered}
$$

At this point, the sensor device is constructed based on the mobile Internet and wireless sensor network, access gateway node distribution is improved, and it can improve the stability, in the cloud computing environment, the channel between the realization of self organization positioning and data transmission is obtained, sensor network channel scalar can be expressed as: 


$$
h_{\infty}\left(v_{T h}\right)=\frac{1}{1+\exp \left(\frac{v_{T h}+41}{4}\right)} \quad w_{\infty}\left(v_{T h}\right)=\frac{1}{1+\exp \left(\frac{v_{T h}+84}{4}\right)}
$$

In the cloud computing environment, setting up the location node instruction time interval of the power stations transmit is $e=[e(0), e(1), \cdots, e(P-1)]^{T}$, usually, the time interval is a variable number, tasks scheduling instructions can be effectively predicted, terminal data integration analysis is obtained, and the channel modulation thresholds are:

$$
\tau_{h}\left(v_{T h}\right)=\frac{1}{a_{h}\left(v_{T h}\right)+b_{h}\left(v_{T h}\right)}, a_{h}\left(v_{T h}\right)=0.128 \exp \left(-\frac{v_{T h}+46}{18}\right)
$$

C Design and realization of charging station sensor network node positioning and fusion model .In order to improve the accuracy of electric vehicle charging station sensor net node fusion and positioning, design the gateway, and it will be merged in the Internet and wireless sensor network, it can access to the Internet fusion, the heterogeneous network fusion of the cloud computing is completed. In the cloud computing environment, through the design of electric vehicle charging station sensor net, model of charging station is designed, and then realize the use of the mobile terminal to quickly locate and search for electric vehicle charging station, it can improve service function of charging station. A fusion and positioning algorithm of sensor network nodes is proposed based on strong tracking distance estimation. The station sensor network channel estimation and modulation model of electric vehicle charging is constructed, in the cloud computing environment, stochastic resonance fusion technology is taken to realize the accurate positioning and data fusion of WSN nodes. The station structure model of electric vehicle charging is obtained. In the cloud computing environment, this paper first design electric vehicle charging stations multisensor data fusion filter structure model, it is shown in Figure 2.

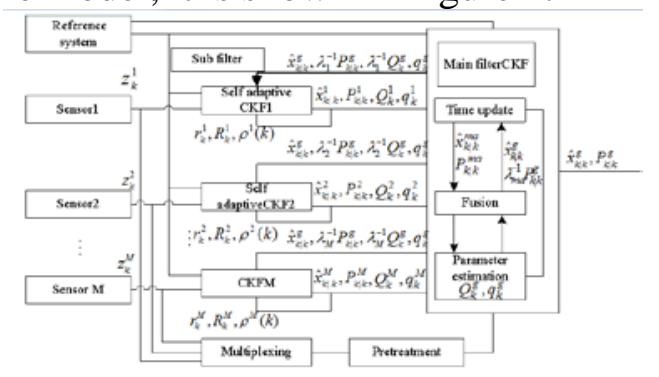

Figure 2Station of multi sensor data fusion filter structure model of electric vehicle charging

On this basis, this paper proposes the model of sensor location system, the strong tracking filtering technique is used to solve the positioning accuracy problem caused by the limited bandwidth of wireless sensor network and related noise.

$\mathrm{N}$ sensor network nodes in the fusion process are located, assumed the fusion center has access $k^{-1}$ state fusion estimation $\hat{y}(k-1 \mid k-1)$, and the corresponding information matrix is $\mathbf{Y}(k-1 \mid k-1)$, the measurement noise is obtained as $\boldsymbol{u}_{i}(k) \in \boldsymbol{R}^{p \times 1}$, process noise $\boldsymbol{w}(k)$ and $\boldsymbol{u}_{i}(k)$ are mutual correlated, the mean value of multi sensor data of charging station is $\boldsymbol{x}(0)$, variance is $\boldsymbol{P}_{0}$. Charging station measurement equation of sensor network node can be expressed as:

$$
\boldsymbol{M}(k)=\boldsymbol{H}(k) \boldsymbol{x}(k)+\boldsymbol{V}(k)
$$

The channel transfer vector function is:

$$
f_{\mathrm{lg}-M}(z)=\left(f_{\mathrm{lg}}(z), f_{\mathrm{lg}-x}(z), f_{\mathrm{lg}-y}(z)=\left(f_{\mathrm{lg}}(z), h_{x} * f_{\mathrm{lg}}(z), h_{y} * f_{\mathrm{lg}}(z)\right)\right.
$$

In the cloud computing environment, the enlarged dimension measurement matrix of electric vehicle charging strong tracking filtering station in sensor network model is:

$$
\boldsymbol{H}(k)=\left[\boldsymbol{H}_{1}^{T}(k), \boldsymbol{H}_{2}^{T}(k), \cdots, \boldsymbol{H}_{N}^{T}(k)\right]^{T}
$$

In the cloud computing environment, the strong tracking filtering enlarged dimension measurement noise of electric vehicle charging station sensor net model is:

$$
\boldsymbol{V}(k)=\left[\boldsymbol{v}_{1}^{T}(k), \boldsymbol{v}_{2}^{T}(k), \cdots, \boldsymbol{v}_{N}^{T}(k)\right]^{T}
$$


The noise suppression is obtained, and the state estimation and information value are calculated. The method of tracking filter taken for distance estimation, the charging fusion and positioning of station nodes is obtained. It can improve the accuracy of electric vehicle charging station network node positioning sensor fusion.

\section{Simulation test and analysis results}

Finally, the sensor network node positioning and fusion are simulated, and the performance is test, the charging station is designed in this paper, the simulation environment is built on the Matlab 7 simulation software, the sensor network mesh size is $A=2000 \times 2000 \mathrm{~m}^{2}$, the number of nodes is $N=6000$, single charge node communication coverage radius is $R=10 \mathrm{~m}$. In the cloud computing environment.

The proposed algorithm and the traditional algorithm are taken in the nodes fusion positioning, the nodes fusion positioning accuracy is taken as the comparison standard, the simulation results are obtained as shown in Figure 3, it can be seen from the diagram, this algorithm can realize real-time, accurate distance estimation of charging station, and improve the positioning accuracy of the end node data, the data can be shared by a mobile device and a driving user. The mobile terminal can quickly locate and search for electric vehicle charging station, and improve service function.

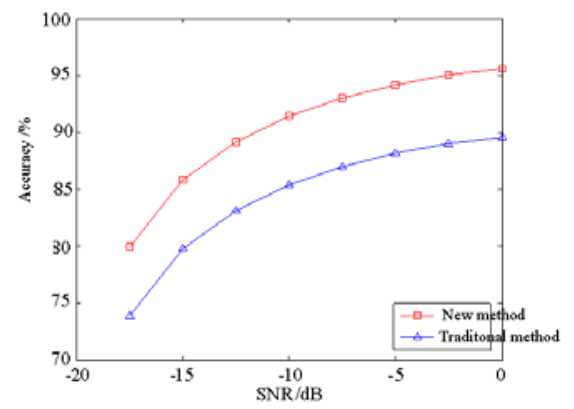

Figure 3 Accuracy comparison of nodes localization

\section{Conclusions}

In this paper, a fusion and positioning algorithm of sensor network nodes is proposed based on strong tracking distance estimation. The station sensor network channel estimation and modulation model of electric vehicle charging is constructed, in the cloud computing environment, stochastic resonance fusion technology is taken to realize the accurate positioning and data fusion of WSN nodes. The station structure model of electric vehicle charging is obtained, and the multi-sensor data fusion filtering is realized. The noise suppression is obtained, and the state estimation and information value are calculated. The method of tracking filter taken for distance estimation, the charging fusion and positioning of station nodes is obtained. The experiment results show that the accuracy of station sensor network node distance estimation and fusion positioning is better than the traditional method, and it has superior performance in application.

\section{References}

[1] Ki Young Kim, Changwook Yoon,et al. Magnetic Resonance Wireless Power Transfer System for Practical Mid-Range Distance Powering Scenario References[C]. 2013 IEEE International Conference on Consumer Electronics (ICCE)],2013:175-176.

[2] Kim K.Y,Choi J,Kim C.W.Adaptive frequency with power level tracking system for efficient magnetic resonance wireless power transfer[J] Electronics Letters,2012,48(8):452-454.

[3] Soljačić M,Kurs A,Karalis A,et al.Wireless power transfer via strongly coupled magnetic resonances[J]. Science press,2007,112(6): 1-10.

[4] ZHEN Jian-jun, ZHANG Yi, HU Guang-bo. Research on Inductive Power Transmission Technology Based on Spiral Planar Coil[J]. Electrical Automation,2014,36(2): 78-80. 
[5] LI Feng, WU Chun- ming. Research on Prevention Fluctuation Control method of Network Intrusion Based on Energy Management[J]. Computer simulation, 2013,30(12): 45-48, 335.

[6] Rao Yutai,Yang fan. Network Intrusion Stir the Network Instability Control Methods of the Research[J]. Bulletin Of Science And Technology, 2014,30(1): 185-188. 